\title{
FIXED-STRUCTURE ROBUST CONTROLLER SYNTHESIS VIA DECENTRALIZED STATIC OUTPUT FEEDBACK
}

\author{
R. SCOTT ERWIN ${ }^{1}$, ANDREW G. SPARKS ${ }^{2}$ AND DENNIS S. BERNSTEIN ${ }^{1 *}$ \\ ${ }^{1}$ Department of Aerospace Engineering, The University of Michigan, Ann Arbor, MI 48109-2118, U.S.A. \\ ${ }^{2}$ WL/FIGC, Building 146, 2210 Eighth Street, Suite 21, Wright-Patterson Air Force Base, OH 45433-7531, U.S.A.
}

\begin{abstract}
SUMMARY
This paper describes and illustrates a unified methodology for robust, fixed-structure controller synthesis. The approach is based upon direct fixed-structure controller synthesis using a decentralized static output feedback formulation as a general framework for representing a large class of controller structures. Scaled Popov bounds for the real structured singular value are used to account for real parameter uncertainty and provide the means for optimizing a worst-case $\mathscr{H}_{2}$ cost bound with respect to the free parameters of the controller. Quasi-Newton optimization algorithms are used to solve the resulting numerical optimization problem. Initial stability multiplier and scaling matrices needed in scaled Popov synthesis are obtained by solving an LMI feasibility problem. Using both centralized and decentralized controller structures, numerical results are obtained for a 16th-order acoustic duct model with uncertain damped natural frequencies and for a two-dimensional beam-spring model with uncertain actuator locations. (C) 1998 John Wiley \& Sons, Ltd.
\end{abstract}

Key words: robust control; decentralized control; reduced order control

\section{INTRODUCTION}

The ability to efficiently synthesize MIMO controllers that meet realistic performance criteria, are robust to real and complex structured perturbations, and can be implemented in a practical way remains one of the principal objectives of modern control theory. This paper describes and illustrates an approach that utilizes the following theoretical and computational techniques:

1. Direct reduced-order controller synthesis via fixed-structure techniques. This approach, which avoids indirect model reduction techniques for plant or controller reduction, ${ }^{1-3}$ provides a direct path from a high-order plant model to a low-order controller. ${ }^{4}$

2. Decentralized static output feedback controller architecture for fixed-structure controller synthesis. In the spirit of previous work on optimal control subject to controller architecture constraints, ${ }^{5,6}$ this problem formulation provides a unified framework for capturing a large class of controller structures, including both static and dynamic controllers in centralized and decentralized architectures with order constraints. ${ }^{7-9}$

This paper was recommended for publication by editor S. Banda

\footnotetext{
* Correspondence to: D. S. Bernstein, Department of Aerospace Engineering, The University of Michigan, Ann Arbor, MI 48109-2118, U.S.A.
} 
3. Scaled Popov bounds for real structured singular value synthesis. In order to account for structured uncertainty that is modelled as constant real parameters, scaled Popov bounds for the real structured singular value are employed. ${ }^{10-12}$ These bounds provide the means for optimizing a worst-case $\mathscr{H}_{2}$ cost bound with respect to the controller parameters. The cost gradients are used by the optimization algorithm to compute controller gains, thus providing a technique for real structured singular value synthesis ${ }^{13,14}$ that does not require curve fitting. ${ }^{15,16}$

4. Convex feasibility of linear matrix inequalities (LMIs). Initialization of stability multiplier and scaling matrices for real structured singular value synthesis is formulated as an optimization problem subject to an LMI constraint. ${ }^{17}$ This problem is convex and can be solved using interior-point methods. ${ }^{14,18-20}$

5. Quasi-Newton optimization algorithms. Quasi-Newton optimization algorithms ${ }^{21,22}$ are used to solve the parameter optimization problem as in References 23-26.

These ideas and techniques are combined to provide a unified methodology for robust fixed-architecture controller synthesis. This paper does not attempt to address the fundamental and difficult problem of determining the existence or uniqueness of stabilizing controllers of a given order, structure and robustness. Rather, the goal is to characterize such controllers when they do exist, and develop a practical approach for computing them in these cases.

Although our approach is based upon fixed-structure optimization, a more common approach is to first obtain high-order controllers which are then reduced to a manageable order for implementation. ${ }^{1-3}$ Although this approach often yields acceptable results, extensions to more specialized problems such as decentralized controller synthesis, which is addressed by our approach, are not available.

Alternative methods for fixed-structure optimization have also been developed. In certain cases, homotopy algorithms ${ }^{6,27-29}$ are numerically more efficient than quasi-Newton methods and do not require an initial controller of the desired structure. Yet another technique for fixed-structure optimization involves bilinear matrix inequalities. ${ }^{30}$

The contents of the paper are as follows. Section 2 reviews the decentralized static output feedback control problem, while Section 3 demonstrates how this formulation can capture a large class of controller structures. In Section 4 , the $\mathscr{H}_{2}$-optimal control problem is reviewed, and gradient expressions for $\mathscr{H}_{2}$-optimal decentralized static output feedback controller synthesis are developed. These results are then extended to robust control in Section 5 where gradients for decentralized static output feedback synthesis using the scaled Popov performance criterion are derived. Section 6 describes the optimization algorithm and discusses initialization issues, while Section 7 provides examples of both centralized and decentralized robust controller synthesis. Section 8 contains a discussion of the paper's results and some conclusions.

\section{THE DECENTRALIZED STATIC OUTPUT FEEDBACK FRAMEWORK}

This section reviews the decentralized static output feedback problem formulation for fixedstructure controller synthesis. Consider the $(m+p+1)$ vector input, $(m+p+1)$ vector output decentralized system shown in Figure 1. Furthermore, define

$$
u=\left[\begin{array}{c}
u_{1} \\
\vdots \\
u_{m}
\end{array}\right], y=\left[\begin{array}{c}
y_{1} \\
\vdots \\
y_{m}
\end{array}\right], d=\left[\begin{array}{c}
d_{1} \\
\vdots \\
d_{p}
\end{array}\right], e=\left[\begin{array}{c}
e_{1} \\
\vdots \\
e_{p}
\end{array}\right]
$$




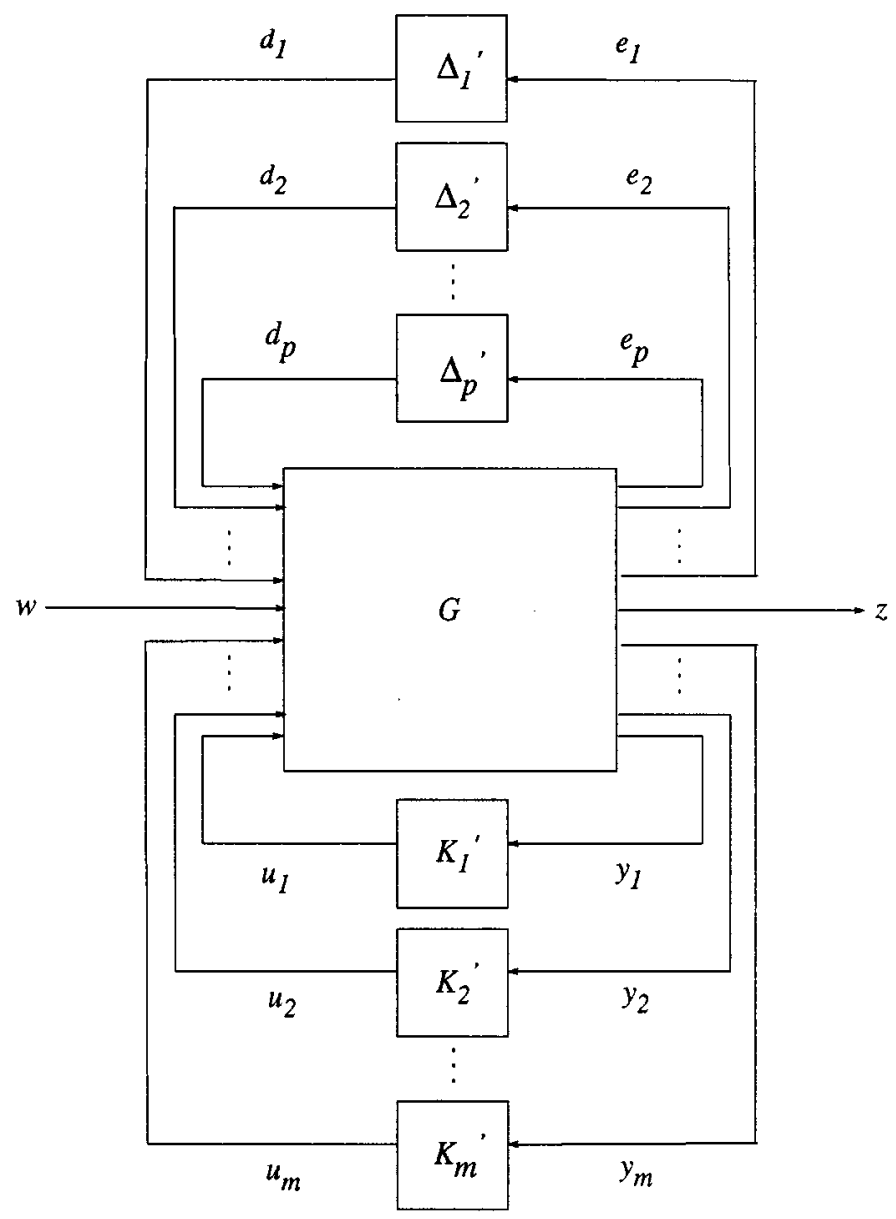

Figure 1. Decentralized static output feedback framework

and let $G(s)$ have the realization

$$
G(s) \sim\left[\begin{array}{c|c:c:r}
\mathscr{A} & \mathscr{B}_{u} & \mathscr{B}_{d} & \mathscr{B}_{w} \\
\hline \mathscr{C}_{y} & \mathscr{D}_{y u} & \mathscr{D}_{y d} & \mathscr{D}_{y w} \\
\hdashline \mathscr{C}_{e} & -\mathscr{D}_{e u} & -\mathscr{D}_{e d} & -\mathscr{D}_{\text {ew }} \\
\hdashline \mathscr{C}_{z} & \mathscr{D}_{z u} & \mathscr{D}_{z d} & \mathscr{D}_{z w}
\end{array}\right]
$$

The realization of $G(s)$ represents the dynamics

$$
\dot{x}=\mathscr{A} x+\mathscr{B}_{u} u+\mathscr{B}_{d} d+\mathscr{B}_{w} w
$$

with measurements

$$
y=\mathscr{C}_{y} x+\mathscr{D}_{y u} u+\mathscr{D}_{y d} d+\mathscr{D}_{y w} w
$$


model error outputs

$$
e=\mathscr{C}_{e} x+\mathscr{D}_{e u} u+\mathscr{D}_{e d} d+\mathscr{D}_{e w} w
$$

and performance variables

$$
z=\mathscr{C}_{z} x+\mathscr{D}_{z u} u+\mathscr{D}_{z d} d+\mathscr{D}_{z w} w
$$

Plant uncertainty is represented as decentralized static output feedback by the relations

$$
d_{i}=\Delta_{i}^{\prime} e_{i}, \quad i=1, \ldots, p
$$

where the uncertain matrices $\Delta_{i}^{\prime}$ are not necessarily distinct. To represent decentralized static output feedback control with possibly repeated gains, we consider

$$
u_{i}=\mathscr{K}_{i}^{\prime} y_{i}, \quad i=1, \ldots, m
$$

where the matrices $\mathscr{K}_{i}^{\prime}$ are not necessarily distinct. Reordering the variables in (7) and (8) if necessary, we can rewrite (7), (8) as

$$
\begin{gathered}
d=\Delta e \\
u=\mathscr{K} y
\end{gathered}
$$

where $\Delta$ and $\mathscr{K}$ belong to the sets

$$
\begin{gathered}
\Delta \triangleq\left\{\Delta: \Delta=\text { block-diag }\left(I_{\psi 1} \otimes \Delta_{1}, \ldots, I_{\psi q} \otimes \Delta_{q}\right)\right\} \\
\mathscr{U} \triangleq\left\{\mathscr{K}: \mathscr{K}=\text { block-diag }\left(I_{\phi 1} \otimes \mathscr{K}_{1}, \ldots, I_{\phi v} \otimes \mathscr{K}_{v}\right)\right\}
\end{gathered}
$$

where $q$ is the number of distinct uncertainties $\Delta_{i} \in \mathscr{R}^{l_{i} \times g_{i}}, \psi_{i}$ is the number of repetitions of uncertainty $\Delta_{i}, v$ is the number of distinct gains $\mathscr{K}_{i} \in \mathscr{R}^{r_{i} \times c_{i}}$ and $\phi_{i}$ is the number of repetitions of gain $\mathscr{K}_{i}$. Here $I_{n}$ represents the $n \times n$ identity matrix. Note that $\Delta_{1}, \ldots, \Delta_{q}$ and $\mathscr{K}_{1}, \ldots, \mathscr{K}_{v}$ are not necessarily square matrices, and that

$$
\sum_{i=1}^{q} \psi_{i}=p, \quad \sum_{i=1}^{v} \phi_{i}=m
$$

For convenience, define

$$
L_{\mathscr{K}} \triangleq I-\mathscr{D}_{\text {yu }} \mathscr{K}
$$

We will make the following assumption for the remainder of the paper.

\section{Assumption 2.1}

The realization (2) satisfies

$$
\begin{gathered}
\mathscr{D}_{\text {ed }}=0 \\
\operatorname{det} L_{\mathscr{K}} \neq 0 \text { for all } \mathscr{K} \in \mathscr{U}
\end{gathered}
$$

Condition (14) implies that the uncertain real parameters appear affinely in the realization of the closed-loop system, while (15) is a well-posedness condition on the algebraic loops that arise from the feedback configuration.

For robust performance analysis, we will be concerned with properties of the system $M(s)$, which is obtained by closing the control feedaback loops around $G(s)$, yielding

$$
\left[\begin{array}{l}
e \\
z
\end{array}\right]=M\left[\begin{array}{l}
d \\
w
\end{array}\right]
$$




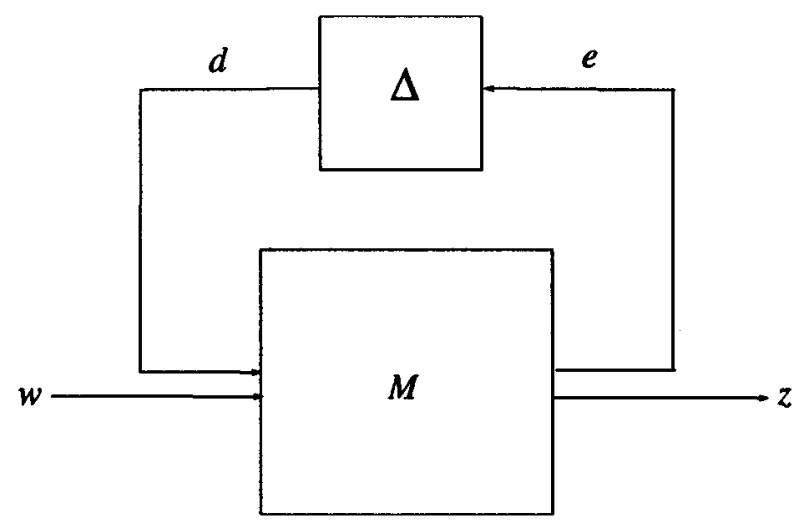

Figure 2. Closed-loop configuration

This configuration is shown in Figure 2. Under Assumption 2.1, the dynamics of this system are given by

$$
\begin{aligned}
& \dot{x}=\tilde{A} x+\widetilde{B}_{d} d+\widetilde{B}_{w} w \\
& e=\widetilde{C}_{e} x+\widetilde{D}_{e d} d+\widetilde{D}_{e w} w \\
& z=\widetilde{C}_{z} x+\widetilde{D}_{z d} d+\widetilde{D}_{z w} w
\end{aligned}
$$

where

$$
\begin{array}{lll}
\tilde{A} \triangleq \mathscr{A}+\mathscr{B}_{u} \mathscr{K} L_{\mathscr{K}}^{-1} \mathscr{C}_{y}, & \widetilde{B}_{d} \triangleq \mathscr{B}_{d}+\mathscr{B}_{u} \mathscr{K} L_{\mathscr{K}}^{-1} \mathscr{D}_{y d}, & \widetilde{B}_{w} \triangleq \mathscr{B}_{w}+\mathscr{B}_{u} \mathscr{K} L_{\mathscr{K}}^{-1} \mathscr{D}_{y w} \\
\tilde{C}_{z} \triangleq \mathscr{C}_{z}+\mathscr{D}_{z u} \mathscr{K} L_{\mathscr{K}}^{-1} \mathscr{C}_{y}, & \widetilde{D}_{z d} \triangleq \mathscr{D}_{z d}+\mathscr{D}_{z u} \mathscr{K} L_{\mathscr{K}}^{-1} \mathscr{D}_{y d}, & \widetilde{D}_{z w} \triangleq \mathscr{D}_{z w}+\mathscr{K} L_{\mathscr{K}}^{-1} \mathscr{D}_{y w} \\
\tilde{C}_{e} \triangleq \mathscr{C}_{e}+\mathscr{D}_{e u} \mathscr{K} L_{\mathscr{K}}^{-1} \mathscr{C}_{y}, & \tilde{D}_{e d} \triangleq \mathscr{D}_{e d}+\mathscr{D}_{e u} \mathscr{K} L_{\mathscr{K}}^{-1} \mathscr{D}_{y d}, & \tilde{D}_{e w} \triangleq \mathscr{D}_{e w}+\mathscr{D}_{e u} \mathscr{K} L_{\mathscr{K}}^{-1} \mathscr{D}_{y w}
\end{array}
$$

Thus $M(s)$ has a realization

$$
M(s) \sim\left[\begin{array}{c|c:c}
\tilde{A} & \tilde{B}_{d} & \tilde{B}_{w} \\
\hline \tilde{C}_{e} & \tilde{D}_{e d} & \tilde{D}_{e w} \\
\hdashline \tilde{C}_{z} & \tilde{D}_{z d} & \tilde{D}_{z w}
\end{array}\right]
$$

For each uncertainty $\Delta \in \Delta$, the closed-loop transfer function $\widetilde{G}_{z w, \Delta}(s)$ thus has the realization

$$
\tilde{G}_{z w, \Delta}(s) \sim\left[\begin{array}{c|c}
\tilde{A}+\tilde{B}_{d} \Delta \tilde{C}_{e} & \widetilde{B}_{w}+\widetilde{B}_{d} \Delta \tilde{D}_{e w} \\
\hline \tilde{C}_{z}+\widetilde{D}_{z d} \Delta \tilde{C}_{e} & \widetilde{D}_{z w}+\widetilde{D}_{z d} \Delta \tilde{D}_{e w}
\end{array}\right]
$$

while the nominal closed-loop transfer function $\widetilde{G}_{z w}(s)=\widetilde{G}_{z w, 0}(s)$ (obtained by letting $\Delta=0$ ) has the realization

$$
\widetilde{G}_{z w}(s) \sim\left[\begin{array}{c|c}
\tilde{A} & \widetilde{B}_{w} \\
\hline \tilde{C}_{z} & \widetilde{D}_{z w}
\end{array}\right]
$$




\section{PROBLEM FORMULATION IN DECENTRALIZED STATIC OUTPUT FEEDBACK FRAMEWORK}

In this section we derive the equivalent decentralized static output feedback representations for several fixed-structure compensation problems. A nominal plant of the form

$$
\begin{aligned}
\dot{x}_{p} & =A x_{p}+B \hat{u}+F_{1} w \\
\hat{y} & =C x_{p}+D \hat{u}+F_{2} w \\
z & =E_{1} x_{p}+E_{2} \hat{u}+E_{0} w
\end{aligned}
$$

will be considered throughout this section unless otherwise specified. In each subsection we consider a specific controller structure and present the equivalent decentralized static output feedback realization. Sections 3.1-3.4 consider the nominal system only, while Sections 3.5 and 3.6 consider an uncertain plant. It is easily verified that Assumption 2.1 is satisfied for all of these examples.

\subsection{Centralized proper dynamic compensation}

First consider an $n_{c}$ th-order proper compensator

$$
\begin{gathered}
\dot{x}_{\mathrm{c}}=A_{\mathrm{c}} x_{\mathrm{c}}+B_{\mathrm{c}} \hat{y} \\
\hat{u}=C_{\mathrm{c}} x_{\mathrm{c}}+D_{\mathrm{c}} \hat{y}
\end{gathered}
$$

Assuming $L=I-D D_{\mathrm{c}}$ is non-singular, a realization of the closed-loop system $M(s)$ consisting of (23)-(27) is given by

$$
M(s) \sim\left[\begin{array}{cc|c:c}
A+B D_{\mathrm{c}} L^{-1} C & B C_{\mathrm{c}}+B D_{\mathrm{c}} L^{-1} D C_{\mathrm{c}} & 0 & F_{1}+B D_{\mathrm{c}} L^{-1} F_{2} \\
B_{\mathrm{c}} L^{-1} C & A_{\mathrm{c}}+B_{\mathrm{c}} L^{-1} D C_{\mathrm{c}} & 0 & B_{\mathrm{c}} L^{-1} F_{2} \\
\hline 0 & 0 & 0 & 0 \\
\hdashline E_{1}+E_{2} D_{\mathrm{c}} L^{-1} C & E_{2} C_{\mathrm{c}}+E_{2} D_{\mathrm{c}} L^{-1} D C_{\mathrm{c}} & 0 & E_{0}+E_{2} D_{\mathrm{c}} L^{-1} F_{2}
\end{array}\right]
$$

This system can be written as decentralized static output feedback with $m=v=\phi_{1}=1, G(s)$ given by

$$
G(s) \sim\left[\begin{array}{cc|cc:c:c}
A & 0 & 0 & B & 0 & F_{1} \\
0 & 0 & I & 0 & 0 & 0 \\
\hline 0 & I & 0 & 0 & 0 & 0 \\
C & 0 & 0 & D & 0 & F_{2} \\
\hdashline 0 & 0 & 0 & 0 & 0 & 0 \\
\hdashline E_{1} & 0 & 0 & E_{2} & 0 & E_{0}
\end{array}\right]
$$

and $\mathscr{K}$ denoting the partitioned matrix

$$
\mathscr{K}=\left[\begin{array}{ll}
A_{\mathrm{c}} & B_{\mathrm{c}} \\
C_{\mathrm{c}} & D_{\mathrm{c}}
\end{array}\right]
$$


In this case, $L_{\mathscr{K}}$ is given by

$$
L_{\mathscr{K}}=\left[\begin{array}{cc}
I & 0 \\
-D C_{\mathrm{c}} & I-D D_{\mathrm{c}}
\end{array}\right]
$$

which is non-singular under the assumption that $L$ is non-singular. Furthermore, from (29), we have that $\mathscr{D}_{e d}=0$. Thus, Assumption 2.1 is satisfied.

\subsection{Centralized strictly proper dynamic compensation}

Consider an $n_{\mathrm{c}}$ th-order strictly proper compensator having the realization

$$
\begin{gathered}
\dot{x}_{\mathrm{c}}=A_{\mathrm{c}} x_{\mathrm{c}}+B_{\mathrm{c}} \hat{y} \\
\hat{u}=C_{\mathrm{c}} x_{\mathrm{c}}
\end{gathered}
$$

A realization for the closed-loop system $M(s)$ consisting of (23)-(25), (32) and (33) is given by

$$
M(s) \sim\left[\begin{array}{cc|c:c}
A & B C_{\mathrm{c}} & 0 & F_{1} \\
B_{\mathrm{c}} C & A_{\mathrm{c}}+B_{\mathrm{c}} D C_{\mathrm{c}} & 0 & B_{\mathrm{c}} F_{2} \\
\hline 0 & 0 & 0 & 0 \\
\hdashline E_{1} & E_{2} C_{\mathrm{c}} & 0 & E_{0}
\end{array}\right]
$$

This system can be written as decentralized static output feedback with $m=v=3, \phi_{1}=\phi_{2}=$ $\phi_{3}=1, G(s)$ given by

$$
G(\mathrm{~s}) \sim\left[\begin{array}{cc|ccc:c:c}
A & 0 & 0 & 0 & B & 0 & F_{1} \\
0 & 0 & I & I & 0 & 0 & 0 \\
\hline 0 & I & 0 & 0 & 0 & 0 & 0 \\
C & 0 & 0 & 0 & D & 0 & F_{2} \\
0 & I & 0 & 0 & 0 & 0 & 0 \\
\hdashline 0 & 0 & 0 & 0 & 0 & 0 & 0 \\
\hdashline E_{1} & 0 & 0 & 0 & E_{2} & 0 & E_{0}
\end{array}\right]
$$

and $\mathscr{K}$ denoting the block-diagonal matrix

$$
\mathscr{K}=\text { block-diag }\left(A_{\mathrm{c}}, B_{\mathrm{c}}, C_{\mathrm{c}}\right)
$$

This yields

$$
L_{\mathscr{K}}=\left[\begin{array}{ccc}
I & 0 & 0 \\
0 & I & -D C_{\mathrm{c}} \\
0 & 0 & I
\end{array}\right]
$$

which is non-singular. From (35), we have $\mathscr{D}_{e d}=0$, and thus Assumption 2.1 is satisfied.

\subsection{Decentralized strictly proper dynamic compensation}

An $m$-channel decentralized dynamic compensator with dynamic compensator of order $n_{c i}$ in the $i$ th channel will be referred to as $\left(n_{c 1}, n_{c 2}, \ldots, n_{c m}\right)$ th order. For example, a two-channel decentralized compensator with a second-order controller in the first channel and a fourth-order compensator in the second channel is of $(2,4)$ th order. 
Let $\hat{u}$ and $\hat{y}$ each be partitioned into two vector channels by $\hat{u}=\left[\hat{u}_{1}^{\mathrm{T}} \hat{u}_{2}^{\mathrm{T}}\right]^{\mathrm{T}}, \hat{y}=\left[\begin{array}{ll}\hat{y}_{1}^{\mathrm{T}} & \hat{y}_{2}^{\mathrm{T}}\end{array}\right]^{\mathrm{T}}$, and rewrite (23)-(25) as

$$
\begin{aligned}
\dot{x} & =A x+B_{1} \hat{u}_{1}+B_{2} \hat{u}_{2}+F_{1} w \\
\hat{y}_{1} & =C_{1} x+D_{11} \hat{u}_{1}+D_{12} \hat{u}_{2}+F_{21} w \\
\hat{y}_{2} & =C_{2} x+D_{21} \hat{u}_{1}+D_{22} \hat{u}_{2}+F_{22} w \\
z & =E_{1} x+E_{21} \hat{u}_{1}+E_{22} \hat{u}_{2}+E_{0} w
\end{aligned}
$$

We consider an $\left(n_{c 1}, n_{c 2}\right)$ th-order, strictly proper compensator

$$
\begin{aligned}
\dot{x}_{\mathrm{c} 1} & =A_{\mathrm{c} 1} x_{\mathrm{c} 1}+B_{\mathrm{c} 1} \hat{y}_{1} \\
\hat{u}_{1} & =C_{\mathrm{c} 1} x_{\mathrm{c} 1} \\
\dot{x}_{\mathrm{c} 2} & =A_{\mathrm{c} 2} x_{\mathrm{c} 2}+B_{\mathrm{c} 2} \hat{y}_{2} \\
\hat{u}_{2} & =C_{\mathrm{c} 2} x_{\mathrm{c} 2}
\end{aligned}
$$

A realization for the closed-loop system $M(s)$ consisting of (38)-(45) is given by

$$
M(s) \sim\left[\begin{array}{ccc|c:c}
A & B_{1} C_{\mathrm{c} 1} & B_{2} C_{\mathrm{c} 2} & 0 & F_{1} \\
B_{\mathrm{c} 1} C_{1} & A_{\mathrm{c} 1}+B_{\mathrm{c} 1} D_{11} C_{\mathrm{c} 1} & B_{\mathrm{c} 1} D_{12} C_{\mathrm{c} 2} & 0 & B_{\mathrm{c} 1} F_{21} \\
B_{\mathrm{c} 2} C_{2} & B_{\mathrm{c} 2} D_{21} C_{\mathrm{c} 1} & A_{\mathrm{c} 2}+B_{\mathrm{c} 2} D_{22} C_{\mathrm{c} 2} & 0 & B_{\mathrm{c} 2} F_{22} \\
\hdashline 0 & 0 & 0 & 0 & 0 \\
\hdashline E_{1} & E_{21} C_{\mathrm{c} 1} & E_{22} C_{\mathrm{c} 2} & 0 & E_{0}
\end{array}\right]
$$

This system can be written as decentralized static output feedback with $m=v=6, \phi_{1}=\phi_{2}=$ $\phi_{3}=\phi_{4}=\phi_{5}=\phi_{6}=1, G(s)$ given by

$$
G(s) \sim\left[\begin{array}{ccc|cccccc:c:c}
A & 0 & 0 & 0 & 0 & B_{1} & 0 & 0 & B_{2} & 0 & D_{1} \\
0 & 0 & 0 & I & I & 0 & 0 & 0 & 0 & 0 & 0 \\
0 & 0 & 0 & 0 & 0 & 0 & I & I & 0 & 0 & 0 \\
\hline 0 & I & 0 & 0 & 0 & 0 & 0 & 0 & 0 & 0 & 0 \\
C_{1} & 0 & 0 & 0 & 0 & D_{11} & 0 & 0 & D_{12} & 0 & D_{21} \\
0 & I & 0 & 0 & 0 & 0 & 0 & 0 & 0 & 0 & 0 \\
0 & 0 & I & 0 & 0 & 0 & 0 & 0 & 0 & 0 & 0 \\
C_{2} & 0 & 0 & 0 & 0 & D_{21} & 0 & 0 & D_{22} & 0 & D_{22} \\
0 & 0 & I & 0 & 0 & 0 & 0 & 0 & 0 & 0 & 0 \\
\hdashline 0 & 0 & 0 & 0 & 0 & 0 & 0 & 0 & 0 & 0 & 0 \\
\hdashline E_{1} & 0 & 0 & 0 & 0 & E_{21} & 0 & 0 & E_{22} & 0 & E_{0}
\end{array}\right]
$$

and $\mathscr{K}$ denoting the block-diagonal matrix

$$
\mathscr{K}=\text { block-diag }\left(A_{\mathrm{c} 1}, B_{\mathrm{c} 1}, C_{\mathrm{c} 1}, A_{\mathrm{c} 2}, B_{\mathrm{c} 2}, C_{\mathrm{c} 2}\right)
$$


In this case,

$$
L_{\mathscr{K}}=\left[\begin{array}{cccccc}
I & 0 & 0 & 0 & 0 & 0 \\
0 & I & -D_{11} C_{\mathrm{c} 1} & 0 & 0 & -D_{12} C_{\mathrm{c} 2} \\
0 & 0 & I & 0 & 0 & 0 \\
0 & 0 & 0 & I & 0 & 0 \\
0 & 0 & -D_{21} C_{\mathrm{c} 1} & 0 & I & -D_{22} C_{\mathrm{c} 2} \\
0 & 0 & 0 & 0 & 0 & I
\end{array}\right]
$$

which is non-singular. From (47), we have $\mathscr{D}_{e d}=0$, and thus Assumption 2.1 is satisfied.

\subsection{Centralized strictly proper dynamic compensation with normal form parametrization}

Consider a centralized strictly proper dynamic compensator (32), (33) with the dynamics matrix parametrized in normal form as

where

$$
A_{\mathrm{c}}=\operatorname{block}-\operatorname{diag}\left(A_{\mathrm{c} 1}, \ldots, A_{\mathrm{c} \beta}\right)
$$

$$
A_{\mathrm{c} i}=\left[\begin{array}{cc}
a_{i} & b_{i} \\
-b_{i} & a_{i}
\end{array}\right], \quad i=1, \ldots, \beta
$$

and where the matrices $B_{\mathrm{c}}$ and $C_{\mathrm{c}}$ are unconstrained. The order of the compensator is thus $2 \beta$. The closed-loop equations in terms of these parameters are somewhat complicated, and thus will not be given explicitly. Defining

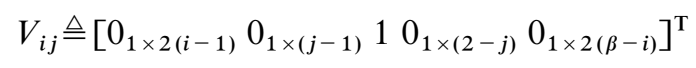

the closed-loop system consisting of (23)-(25) and (32)-(33), with the parametrization (50), can be written as decentralized static output feedback with $m=2 \beta+2, v=\beta+2, \phi_{1}=\phi_{2}=\cdots=$ $\phi_{\beta}=2, \phi_{\beta+1}=\phi_{\beta+2}=1, G(s)$ given by

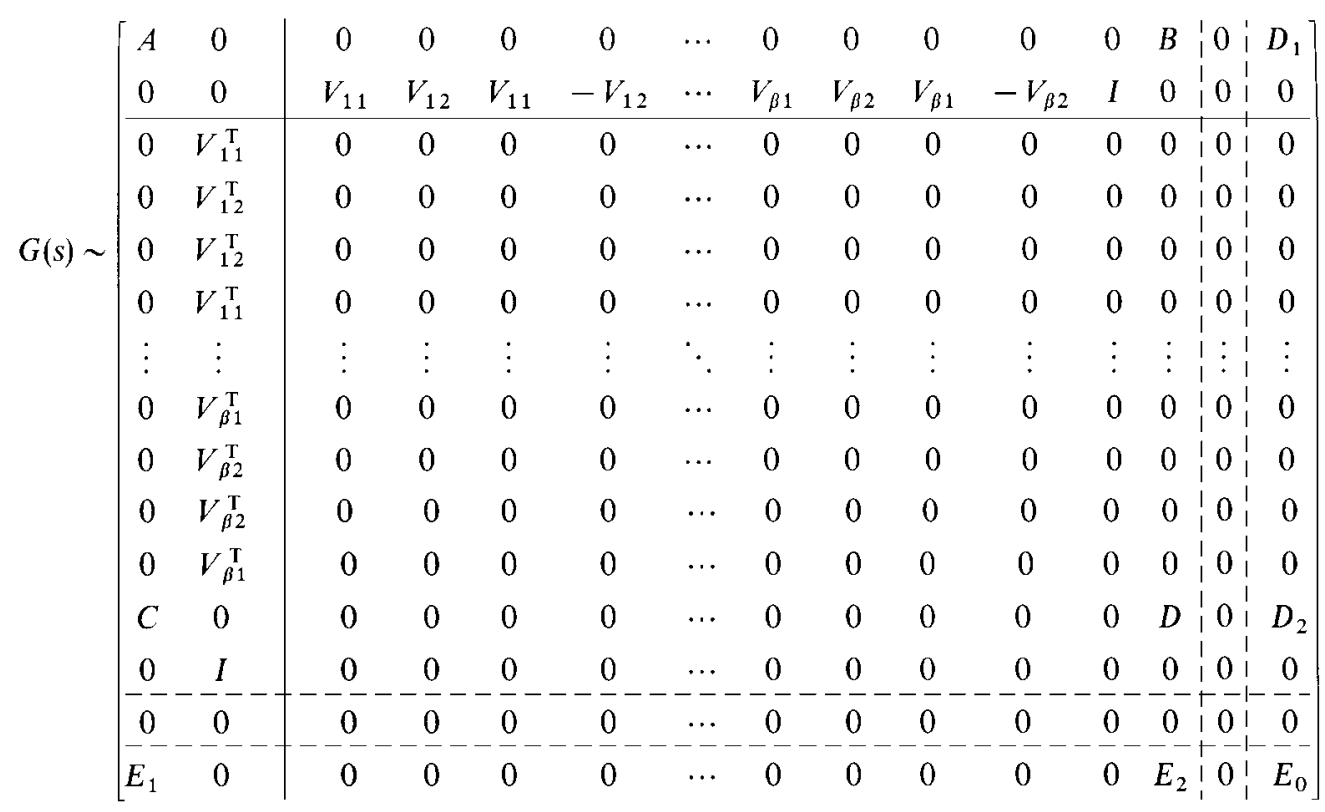


and $\mathscr{K}$ denoting the block-diagonal matrix

$$
\mathscr{K}=\text { block-diag }\left(a_{1}, a_{1}, b_{1}, b_{1}, \ldots, a_{\beta}, a_{\beta}, b_{\beta}, b_{\beta}, B_{\mathrm{c}}, C_{\mathrm{c}}\right)
$$

It can be verified by construction that $L_{\mathscr{K}}$ is non-singular. Furthermore, from (53), we have $\mathscr{D}_{\text {ed }}=0$. Therefore, Assumption 2.1 is satisfied.

This parametrization of the controller involves $n_{\mathrm{c}}+n_{\mathrm{c}}\left(n_{y}+n_{u}\right)$ parameters, compared with the $n_{\mathrm{c}}^{2}+n_{\mathrm{c}}\left(n_{y}+n_{u}\right)$ parameters of the full-matrix parametrization used in Section 3.2. Thus, the number of free variables is reduced by $n_{\mathrm{c}}\left(n_{\mathrm{c}}-1\right)$. This reduction comes at the expense of an implicit constraint on the eigenstructure of the controller, i.e., all eigenvalues of the controller dynamics matrix are assumed to be semi-simple, and all real eigenvalues have even algebraic multiplicity.

\subsection{Centralized strictly proper dynamic compensation with uncertain plant dynamics}

Consider the controller structure given by (32)-(33), and assume that uncertainty in the dynamics of the plant is accounted for by the model

$$
\dot{x}=\left(A+M_{A} \Delta_{A} N_{A}\right) x+B u+F_{1} w
$$

Letting $e=N_{A} x, \Delta=\Delta_{1}=\Delta_{A}$ and $d=\Delta e$, a realization for the closed-loop system $M(s)$ consisting of (24), (25), (32)-(33) and (55) is given by

$$
M(s) \sim\left[\begin{array}{cc|c:c}
A & B C_{\mathrm{c}} & M_{A} & F_{1} \\
B_{\mathrm{c}} C & A_{\mathrm{c}}+B_{\mathrm{c}} D C_{\mathrm{c}} & 0 & B_{\mathrm{c}} F_{2} \\
\hline N_{A} & 0 & 0 & 0 \\
\hdashline E_{1} & E_{2} C_{\mathrm{c}} & 0 & E_{0}
\end{array}\right]
$$

This system can be written as decentralized static output feedback with $m=v=3, \phi_{1}=\phi_{2}=$ $\phi_{3}=1, p=1, \psi_{1}=1, G(s)$ given by

$$
G(s) \sim\left[\begin{array}{cc|ccc:c:c}
A & 0 & 0 & 0 & B & M_{A} & F_{1} \\
0 & 0 & I & I & 0 & 0 & 0 \\
\hline 0 & I & 0 & 0 & 0 & 0 & 0 \\
C & 0 & 0 & 0 & D & 0 & F_{2} \\
0 & I & 0 & 0 & 0 & 0 & 0 \\
\hdashline N_{A} & 0 & 0 & 0 & 0 & 0 & 0 \\
\hdashline E_{1} & 0 & 0 & 0 & E_{2} & 0 & E_{0}
\end{array}\right]
$$

and $\mathscr{K}$ be given by (36). In this case, $L_{\mathscr{K}}$ is given by (37). From (53), we have $\mathscr{D}_{\text {ed }}=0$, and thus Assumption 2.1 is satisfied.

\subsection{Centralized strictly proper dynamic compensation with uncertain input and output matrices}

Consider the controller structure given by (32)-(33), and let the uncertainty in the input and output matrices of the plant be represented by

$$
\dot{x}=A x+\left(B+M_{B} \Delta_{B} N_{B}\right) u+F_{1} w
$$


and

Letting

$$
y=\left(C+M_{C} \Delta_{C} N_{C}\right) x+D u+F_{2} w
$$

$$
e=\left[\begin{array}{c}
N_{B} C_{\mathrm{c}} x_{\mathrm{c}} \\
N_{C} x
\end{array}\right]
$$

$\Delta=$ block-diag $\left(\Delta_{B}, \Delta_{C}\right)$, and $d=\Delta e$, a realization for the closed-loop system $M(s)$ consisting of (25), (32), (33), (58) and (59) is given by

$$
M(s) \sim\left[\begin{array}{cc|cc:c}
A & B C_{\mathrm{c}} & M_{B} & 0 & F_{1} \\
B_{\mathrm{c}} C & A_{\mathrm{c}}+B_{\mathrm{c}} D C_{\mathrm{c}} & 0 & B_{\mathrm{c}} M_{C} & B_{\mathrm{c}} F_{2} \\
\hline 0 & N_{B} C_{\mathrm{c}} & 0 & 0 & 0 \\
N_{C} & 0 & 0 & 0 & 0 \\
\hdashline E_{1} & E_{2} C_{\mathrm{c}} & 0 & 0 & E_{0}
\end{array}\right]
$$

This system can be written as decentralized static output feedback with $m=v=3, \phi_{1}=\phi_{2}=$ $\phi_{3}=1, p=2, \psi_{1}=\psi_{2}=1, G(s)$ given by

$$
G(s) \sim\left[\begin{array}{cc|ccc:cc:c}
A & 0 & 0 & 0 & B & M_{B} & 0 & D_{1} \\
0 & 0 & I & I & 0 & 0 & 0 & 0 \\
\hline 0 & I & 0 & 0 & 0 & 0 & 0 & 0 \\
C & 0 & 0 & 0 & D & 0 & M_{C} & D_{2} \\
0 & I & 0 & 0 & 0 & 0 & 0 & 0 \\
\hdashline 0 & 0 & 0 & 0 & N_{B} & 0 & 0 & 0 \\
N_{C} & 0 & 0 & 0 & 0 & 0 & 0 & 0 \\
\hdashline E_{1} & 0 & 0 & 0 & E_{2} & 0 & 0 & E_{0}
\end{array}\right]
$$

and $\mathscr{K}$ be defined by (36). In this case, $L_{\mathscr{K}}$ is given by (37). From (62), we have $\mathscr{D}_{\text {ed }}=0$, and thus Assumption 2.1 is satisfied.

\section{4. $\mathscr{H}_{2}$-OPTIMAL COMPENSATOR DESIGN}

In this section we consider $\mathscr{H}_{2}$-optimal compensator design for the nominal closed-loop transfer function $\widetilde{G}_{z w}$ with realization (22). To ensure that the $\mathscr{H}_{2}$ norm of $\widetilde{G}_{z w}(s)$ is finite, we invoke the following assumption for the remainder of this section.

\section{Assumption 4.1}

The realization (22) of the nominal closed-loop transfer function $\tilde{G}_{z w}(s)$ satisfies

$$
\tilde{D}_{z w}=0 \text { for all } \mathscr{K} \in \mathscr{U}
$$

As can be seen in Section 3.1 from (28), for centralized, proper dynamic compensation, Assumption 4.1 is equivalent to $E_{0}+E_{2} D_{\mathrm{c}} L^{-1} F_{2}=0$. For the remainder of the problems considered in Section 3, Assumption 4.1 is satisfied if and only if $E_{0}=0$. 
Under Assumption 4.1, it follows from standard results that the $\mathscr{H}_{2}$ norm of $\widetilde{G}_{z w}(s)$ is given by

$$
\mathscr{J}(\mathscr{K})=\left\|\tilde{G}_{z w}\right\|_{2}^{2}=\operatorname{tr} \tilde{P} \tilde{V}
$$

where $\widetilde{P}>0$ satisfies the Lyapunov equation

$$
0=\tilde{A}^{\mathrm{T}} \tilde{P}+\tilde{P} \tilde{A}+\tilde{R}
$$

with $\tilde{R} \triangleq \tilde{C}_{z}^{\mathrm{T}} \tilde{C}_{z}$ and $\tilde{V} \triangleq \widetilde{B}_{w} \widetilde{B}_{w}^{\mathrm{T}}$.

To minimize the cost (64) subject to the constraint (65), define the Lagrangian

$$
\mathscr{L}(\tilde{P}, \mathscr{K})=\operatorname{tr}\left[\tilde{P} \tilde{V}+\widetilde{Q}\left(\tilde{A}^{\mathrm{T}} \tilde{P}+\tilde{P} \tilde{A}+\tilde{R}\right)\right]
$$

where $\widetilde{Q}$ is the matrix Lagrange multiplier. The gradients of $\mathscr{L}$ with respect to $\widetilde{P}$ and $\mathscr{K}_{i}$ are given by

$$
\frac{\partial \mathscr{L}}{\partial \widetilde{P}}=\tilde{A} \tilde{Q}+\tilde{Q} \tilde{A}^{\mathrm{T}}+\tilde{V}
$$

$\frac{\partial \mathscr{L}}{\partial \mathscr{K}_{i}}=2 \sum_{j=1}^{\phi_{i}} Q_{L i j}^{\mathrm{T}}\left(I+\mathscr{D}_{y u}^{\mathrm{T}} L_{\mathscr{K}}^{-\mathrm{T}} \mathscr{K}^{\mathrm{T}}\right)\left(\mathscr{B}_{u}^{\mathrm{T}} \tilde{P} \widetilde{B}_{w} \mathscr{D}_{y w}^{\mathrm{T}}+\mathscr{D}_{z u}^{\mathrm{T}} \tilde{C}_{z} \widetilde{Q} \mathscr{C}_{y}^{\mathrm{T}}+\mathscr{B}_{u}^{\mathrm{T}} \widetilde{P} \widetilde{C^{\mathrm{T}}}{ }_{y}^{\mathrm{T}}\right) L_{\mathscr{K}}^{-\mathrm{T}} Q_{R i j}^{\mathrm{T}} i=1, \ldots, v$

where $Q_{L i j}$ and $Q_{R i j}$ are defined by

$$
Q_{L i j} \triangleq\left[\begin{array}{c}
0_{r_{1} \phi_{1} \times r_{i}} \\
0_{r_{2} \phi_{2} \times r_{i}} \\
\vdots \\
0_{r_{i-1} \phi_{i-1} \times r_{i}} \\
0_{r_{i}(j-1) \times r_{i}} \\
I_{r_{i}} \\
0_{r_{i}\left(\phi_{i}-j\right) \times r_{i}} \\
0_{r_{i+1} \phi_{i+1} \times r_{i}} \\
\vdots \\
0_{r_{v} \phi_{v} \times r_{i}}
\end{array}\right], \quad Q_{R i j} \triangleq\left[\begin{array}{c}
0_{c_{1} \phi_{1} \times c_{i}} \\
0_{c_{2} \phi_{2} \times c_{i}} \\
\vdots \\
0_{c_{i-1} \phi_{i-1} \times c_{i}} \\
0_{c_{i}(j-1) \times c_{i}} \\
I_{c_{i}} \\
0_{c_{i}\left(\phi_{i}-j\right) \times c_{i}} \\
0_{c_{i+1}} \phi_{i+1} \times c_{i} \\
\vdots \\
0_{c_{v} \phi_{v} \times c_{i}}
\end{array}\right]^{\mathrm{T}}
$$

In Reference 9, it was shown that if $\widetilde{P}$ and $\widetilde{Q}$ satisfy both (65) and

$$
0=\tilde{A} \tilde{Q}+\widetilde{Q} \tilde{A}^{\mathrm{T}}+\tilde{V}
$$

then

$$
\frac{\partial \mathscr{J}}{\partial \mathscr{K}_{i}}=\frac{\partial \mathscr{L}}{\partial \mathscr{K}_{i}}, \quad i=1, \ldots, v
$$

\section{DECENTRALIZED SCALED POPOV SYNTHESIS}

In this section we use the scaled Popov criterion ${ }^{11,12,31}$ to synthesize controllers within the decentralized static output feedback framework. The scaled Popov criterion provides robustness guarantees for a norm-bounded, block-structured, real-parameter uncertainty matrix $\Delta$ appearing affinely in the closed-loop dynamics matrix. The following assumption will be invoked for the remainder of this section. 
Assumption 5.1

The realization (21) of the perturbed closed-loop transfer function satisfies

$$
\begin{gathered}
\tilde{D}_{e w}=0 \text { for all } \mathscr{K} \in \mathscr{U} \\
\tilde{D}_{z d}=0 \text { for all } \mathscr{K} \in \mathscr{U}
\end{gathered}
$$

As can be seen from (21), conditions (72) and (73) restrict the class of problems to those in which the uncertain matrix $\Delta$ appears only in the closed-loop dynamics matrix $\tilde{A}+\widetilde{B}_{d} \Delta \widetilde{C}_{e}$. It can be seen that Assumption 5.1 holds for all of the problems considered in Sections 3.5 and 3.6.

The uncertainty $\Delta$ is assumed to be an element of the uncertainty set $\Delta_{\gamma}$ defined by

$$
\Delta_{\gamma} \triangleq\left\{\Delta \in \Delta: \Delta_{i}=\Delta_{i}^{\mathrm{T}} \in \mathscr{R}^{l_{i} \times l_{i}}, i=1, \ldots, q, \sigma_{\max }(\Delta) \leqslant \gamma^{-1}\right\}
$$

where $\sigma_{\max }(\cdot)$ denotes the maximum singular value. As in Section 4, we invoke Assumption 4.1 to ensure that the $\mathscr{H}_{2}$ norm of the closed-loop system is finite. With Assumptions 4.1 and 5.1, the closed-loop transfer function (21) becomes

$$
\widetilde{G}_{z w, \Delta}(s) \sim\left[\begin{array}{c|c}
\tilde{A}+\tilde{B}_{d} \Delta \tilde{C}_{e} & \widetilde{B}_{w} \\
\hline \widetilde{C}_{z} & 0
\end{array}\right]
$$

If the closed-loop system is asymptotically stable for all uncertainty $\Delta \in \boldsymbol{\Delta}_{\gamma}$, then the worst-case closed-loop $\mathscr{H}_{2}$ norm is given by

$$
\sup _{\Delta \in \Delta_{\gamma}}\left\|\tilde{G}_{z w, \Delta}\right\|_{2}^{2}=\sup _{\Delta \in \Delta_{\gamma}} \operatorname{tr} \tilde{P}_{\Delta} \tilde{V}
$$

where $\widetilde{P}_{\Delta}$ satisfies

$$
0=\left(\tilde{A}+\widetilde{B}_{d} \Delta \tilde{C}_{e}\right)^{\mathrm{T}} \tilde{P}_{\Delta}+\tilde{P}_{\Delta}\left(\tilde{A}+\widetilde{B}_{d} \Delta \tilde{C}_{e}\right)+\tilde{R}
$$

For the following result, define the set of symmetric block-diagonal matrices

$$
\begin{gathered}
\mathscr{W} \triangleq\left\{W: W=\text { block-diag }\left(W_{1} \otimes I_{\psi_{1}}, \ldots, W_{q} \otimes I_{\psi_{q}}\right)\right. \\
\left.W_{i}=W_{i}^{\mathrm{T}} \in \mathscr{R}^{l_{i} \times l_{i}}, i=1, \ldots, q\right\}
\end{gathered}
$$

whose elements commute with every $\Delta \in \Delta$, and, for convenience, let

$$
\Gamma \triangleq \gamma Z-W \widetilde{C}_{e} \widetilde{B}_{d}-\widetilde{B}_{d}^{\mathrm{T}} \widetilde{C}_{e}^{\mathrm{T}} W, \quad \mathscr{X} \triangleq \widetilde{B}_{d}^{\mathrm{T}} \tilde{P}+Z \tilde{C}_{e}+W \widetilde{C}_{e} \tilde{A}_{0}
$$

where $\tilde{A}_{0} \triangleq \tilde{A}-\gamma^{-1} \widetilde{B}_{d} \widetilde{C}_{e}$. The following result from Reference 14 will be needed.

\section{Theorem 5.1}

Let $\gamma>0$ and assume $\tilde{A}_{0}$ is asymptotically stable. Furthermore, suppose there exist a positivedefinite matrix $\widetilde{P}$ and matrices $W, Z \in \mathscr{W}$, where $Z>0$, such that $\Gamma>0$ and

$$
0=\tilde{A}_{0}^{\mathrm{T}} \tilde{P}+\tilde{P} \tilde{A}_{0}+\mathscr{X}^{\mathrm{T}} \Gamma^{-1} \mathscr{X}+\tilde{R}
$$

Then $\tilde{G}_{z w, \Delta}$ is asymptotically stable for all $\Delta \in \boldsymbol{\Delta}_{\gamma}$, and

$$
\sup _{\Delta \in \Delta_{\nu}}\left\|\tilde{G}_{z w, \Delta}\right\|_{2}^{2} \leqslant \operatorname{tr}\left(\tilde{P}+2 \gamma^{-1} \tilde{C}_{e}^{\mathrm{T}} W \tilde{C}_{e}\right) \tilde{V}
$$


Using the bound in (81) as an auxiliary cost function and (80) as a constraint, the corresponding Lagrangian is

$$
\mathscr{L}(\tilde{P}, \mathscr{K}, W, Z)=\operatorname{tr}\left(\widetilde{P}+2 \gamma^{-1} \tilde{C}_{e}^{\mathrm{T}} W \tilde{C}_{e}\right) \tilde{V}+\operatorname{tr} \widetilde{Q}\left(\tilde{A}_{0}^{\mathrm{T}} \tilde{P}+\tilde{P} \tilde{A}_{0}+\widetilde{R}+\mathscr{X}^{\mathrm{T}} \Gamma^{-1} \mathscr{X}\right)
$$

The gradients of $\mathscr{L}(\tilde{P}, \mathscr{K}, W, Z)$ are given by

$$
\begin{aligned}
& \frac{\partial \mathscr{L}}{\partial \widetilde{P}}=\tilde{A}_{0} \tilde{Q}+\tilde{Q} \tilde{A}_{0}^{\mathrm{T}}+\tilde{V} \\
& \frac{\partial \mathscr{L}}{\partial \mathscr{K}_{i}}=2 \sum_{j=1}^{\phi_{i}} Q_{L i j}^{\mathrm{T}}\left(I+\mathscr{D}_{y u} L_{\mathscr{K}}^{-\mathrm{T}} \mathscr{K}^{\mathrm{T}}\right)\left[\mathscr{B}_{u}^{\mathrm{T}} \tilde{P} \widetilde{B}_{w} \mathscr{D}_{y w}^{\mathrm{T}}+\mathscr{D}_{z u}^{\mathrm{T}} \widetilde{C}_{z} \widetilde{Q^{T}}{ }_{y}^{\mathrm{T}}+\mathscr{B}_{u}^{\mathrm{T}} \tilde{P} \widetilde{Q} \mathscr{C}_{u}^{\mathrm{T}}\right. \\
& +\mathscr{B}_{u}^{\mathrm{T}} \Gamma^{-1} \mathscr{X}^{\mathrm{T}} Q \widetilde{P} \mathscr{D}_{y d}^{\mathrm{T}}+\mathscr{D}_{e u}^{\mathrm{T}} Z \Gamma^{-1} \mathscr{X}^{\mathrm{T}} Q_{\mathscr{C}_{y}^{\mathrm{T}}}^{\mathrm{T}}+\mathscr{D}_{e u}^{\mathrm{T}} W \Gamma^{-1} \mathscr{X}^{\mathrm{T}} Q \tilde{A}_{0}^{\mathrm{T}} \mathscr{C}_{y}^{\mathrm{T}} \\
& -\gamma^{-1} \mathscr{B}_{u}^{\mathrm{T}} \widetilde{C}_{e}^{\mathrm{T}} W \Gamma^{-1} \mathscr{X}^{\mathrm{T}} Q \widetilde{C}_{e}^{\mathrm{T}} \mathscr{D}_{y d}^{\mathrm{T}}-\gamma^{-1} \mathscr{D}_{e u}^{\mathrm{T}} \widetilde{B}_{d}^{\mathrm{T}} \widetilde{C}_{e}^{\mathrm{T}} W \Gamma^{-1} \mathscr{X}^{\mathrm{T}} Q^{C_{y}^{\mathrm{T}}} \\
& -\mathscr{D}_{e u}^{\mathrm{T}} W \Gamma^{-1} \mathscr{X}^{\mathrm{T}} Q \mathscr{X} \Gamma^{-1} \widetilde{B}_{d}^{\mathrm{T}} \mathscr{C}_{y}^{\mathrm{T}}-\mathscr{B}_{u}^{\mathrm{T}} \tilde{C}_{e}^{\mathrm{T}} W \Gamma^{-1} \mathscr{X}^{\mathrm{T}} Q \mathscr{X} \Gamma^{-1} \mathscr{D}_{y d}^{\mathrm{T}} \\
& +2 \gamma^{-1} \mathscr{D}_{e u}^{\mathrm{T}} W \widetilde{C}_{e} \widetilde{B}_{w} \widetilde{B}_{w}^{\mathrm{T}} \mathscr{C}_{y}^{\mathrm{T}}+2 \gamma^{-1} \mathscr{B}_{u}^{\mathrm{T}} \tilde{C}_{e}^{\mathrm{T}} W \widetilde{C}_{e} \widetilde{B}_{w} \mathscr{D}_{y w}^{\mathrm{T}}-\gamma^{-1} \mathscr{B}_{u}^{\mathrm{T}} \tilde{P} \tilde{Q} \widetilde{C}_{e}^{\mathrm{T}} \mathscr{D}{ }_{y d}^{\mathrm{T}} \\
& \left.-\gamma^{-1} \mathscr{D}_{e u}^{\mathrm{T}} \widetilde{B}_{d}^{\mathrm{T}} \tilde{P} \widetilde{Q} \mathscr{C}_{y}^{\mathrm{T}}+\mathscr{B}_{u}^{\mathrm{T}} \widetilde{C}_{e}^{\mathrm{T}} W \Gamma^{-1} \mathscr{X}^{\mathrm{T}} Q \mathscr{C}_{y}^{\mathrm{T}}\right] L_{\mathscr{K}}^{-\mathrm{T}} Q_{R i j}^{\mathrm{T}}, i=1, \ldots, v \\
& \frac{\partial \mathscr{L}}{\partial W}=\Gamma^{-1} \mathscr{X} \widetilde{Q} \mathscr{X}^{\mathrm{T}} \Gamma^{-1} \widetilde{B}_{d}^{\mathrm{T}} \tilde{C}_{e}^{\mathrm{T}}+\widetilde{B}_{d} \widetilde{C}_{e} \Gamma^{-1} \mathscr{X} \widetilde{Q} \mathscr{X}^{\mathrm{T}} \Gamma^{-1}+\Gamma^{-1} \mathscr{X} \widetilde{Q} \tilde{A}_{0} \widetilde{C}_{e}^{\mathrm{T}} \\
& +\widetilde{C}_{e} \tilde{A}_{0} \widetilde{Q} \mathscr{X}^{\mathrm{T}} \Gamma^{-1}+\gamma^{-1} \widetilde{C}_{e} \tilde{V} \widetilde{C}_{e}^{\mathrm{T}} \\
& \frac{\partial \mathscr{L}}{\partial Z}=\Gamma^{-1} \mathscr{X} \widetilde{Q} \tilde{C}_{e}^{\mathrm{T}}+\tilde{C}_{e} \widetilde{Q} \mathscr{X}^{\mathrm{T}} \Gamma^{-1}-\gamma \Gamma^{-1} \mathscr{X} \widetilde{Q} \mathscr{X}^{\mathrm{T}} \Gamma^{-1}
\end{aligned}
$$

\section{ALGORITHM DESCRIPTION}

Although specialized gradient search algorithms have been developed for control-system parameter optimization, ${ }^{4,23,24,26}$ we utilize the modular, general purpose quasi-Newton algorithms developed by Dennis and Schnabel. ${ }^{21}$ The line-search portions of the code were modified to include a subroutine that decreases the length of the search direction vector until it lies entirely within the set of parameters that yield a stable closed-loop system. This modification enforces closed-loop stability and ensures that the cost function remains defined at every point in the line-search process. Numerical experience indicates that this subroutine is invoked only during the first few iterations of $\mathscr{H}_{2}$-optimal synthesis, while during scaled Popov synthesis the routine tends to be invoked sporadically during the numerical optimization.

Initialization of the optimization algorithm represents a challenging problem for open-loop unstable plants. For open-loop stable plants, however, initialization can be accomplished by using sufficiently low-authority compensators along with an appropriate model reduction technique. ${ }^{32}$ For example, centralized dynamic reduced-order $\mathscr{H}_{2}$-optimal compensators can be initialized by applying balanced truncation to a full-order, Riccati-based LQG controller, where the control weighting matrix in the quadratic cost is chosen to be large relative to the state weighting matrix. These low-authority, full-order controllers can usually be truncated without violating closed-loop stability. For other architectures, this procedure can be implemented sequentially, where successive low-authority controllers are designed for each loop and then 
incorporated into the plant to generate the next loop. These low-authority designs are used to initialize a low-authority optimization algorithm. The optimized controller gains are then used to initialize a modified problem where the control weighting matrix has been reduced to yield a higher authority design. Repeating this procedure yields a sequence of controllers that demonstrate the trade-off between control effort and state cost.

Robust designs can be obtained by an extension of this procedure. An $\mathscr{H}_{2}$-optimal compensator of the appropriate architecture is designed using the above procedure and is used to initialize the scaled Popov problem with a large value of $\gamma$, corresponding to a small uncertainty level. Initial values for the scaling and stability multiplier matrices $W$ and $Z$ must satisfy both $\Gamma>0$ and the constraint equation (80) for a given value of $\gamma$ and a given controller. It was shown in Reference 17 that this problem is equivalent to minimizing tr $\widetilde{P}$ subject to the matrix inequality

$$
\left[\begin{array}{cc}
\tilde{A}_{0}^{\mathrm{T}} \tilde{P}+\tilde{P} \tilde{A}_{0}+\tilde{R} & \mathscr{X}^{\mathrm{T}} \\
\mathscr{X} & -\Gamma
\end{array}\right]<0
$$

Since (87) is affine in the variables $\widetilde{P}, W$ and $Z$, a solution can be computed using interior-point methods. ${ }^{18-20}$ The resulting matrices $W$ and $Z$ can then be used as initial values for the controller gain optimization problem. The synthesis algorithm is applied iteratively with a sequence of decreasing values of the variable $\gamma$, using the controller from the previous iteration as an initial guess for the next optimization problem. This procedure produces a sequence of controllers with increasing levels of robust performance for the given uncertainty structure.

\section{NUMERICAL RESULTS}

To illustrate the techniques described above for fixed-structure synthesis, two numerical examples are considered. First, to demonstrate fixed-structure $\mathscr{H}_{2}$-optimal synthesis and robust controller synthesis for a plant with uncertain dynamics, a 16th-order, two-input/two-output model of an acoustic duct based on the formulation of Reference 33 is considered. To demonstrate robust controller synthesis for a plant with uncertainty in the sensor or actuator positions, a twoinput/two-output model of the two-dimensional rotational and translational dynamics of a rigid beam-spring system is considered. Centralized and decentralized controllers are synthesized for both examples. The data used to construct these examples are provided in the Appendix.

\subsection{Centralized/decentralized strictly proper dynamic $\mathscr{H}_{2}$-optimal compensation}

Centralized and decentralized strictly proper $\mathscr{H}_{2}$-optimal compensators of various orders were synthesized for the acoustic duct model given in the Appendix using the initialization method discussed in Section 6. The resulting trade-off curves generated by varying the control weighting matrix $E_{2}$ are shown in Figures 3 and 4 . As expected, the constraints imposed by the decentralized architecture decrease the achievable performance compared with the centralized designs. Also, note that at high authority levels, the fourth-order centralized designs have converged to a local minimizing solution, as indicated by the fact that the second-order solution achieves a lower state cost than the fourt-order solution for the same normalized control cost.

\subsection{Centralized strictly proper dynamic $\mathscr{H}_{2}$-optimal compensation with normal-form parametrization}

To demonstrate the effect of using an alternative parametrization of the compensator realization, a series of centralized strictly proper eighth-order $\mathscr{H}_{2}$-optimal compensators were designed 


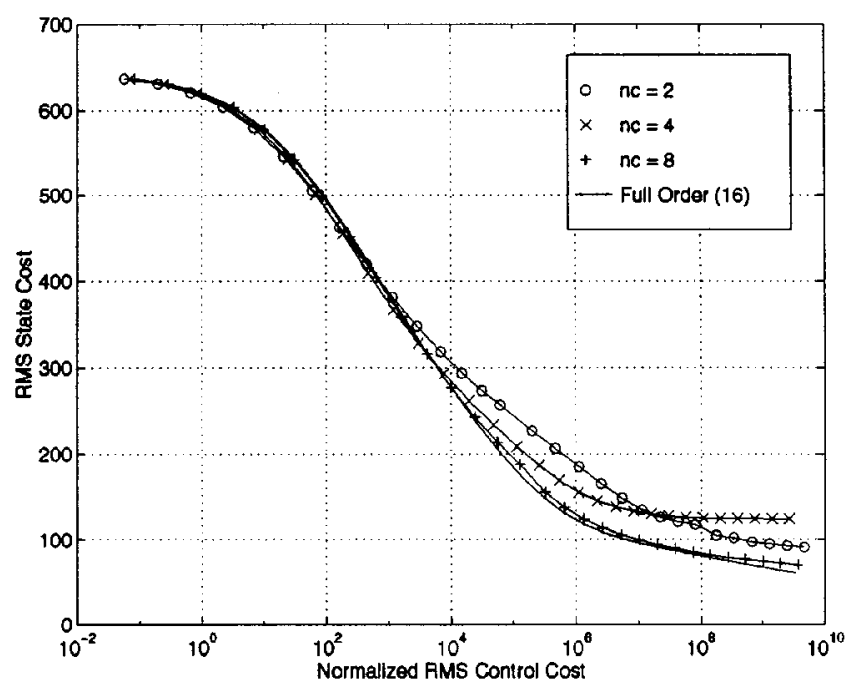

Figure 3. State/control cost trade-off curves for the 16 th-order acoustic duct with centralized $\mathscr{H}_{2}$-optimal dynamic compensation

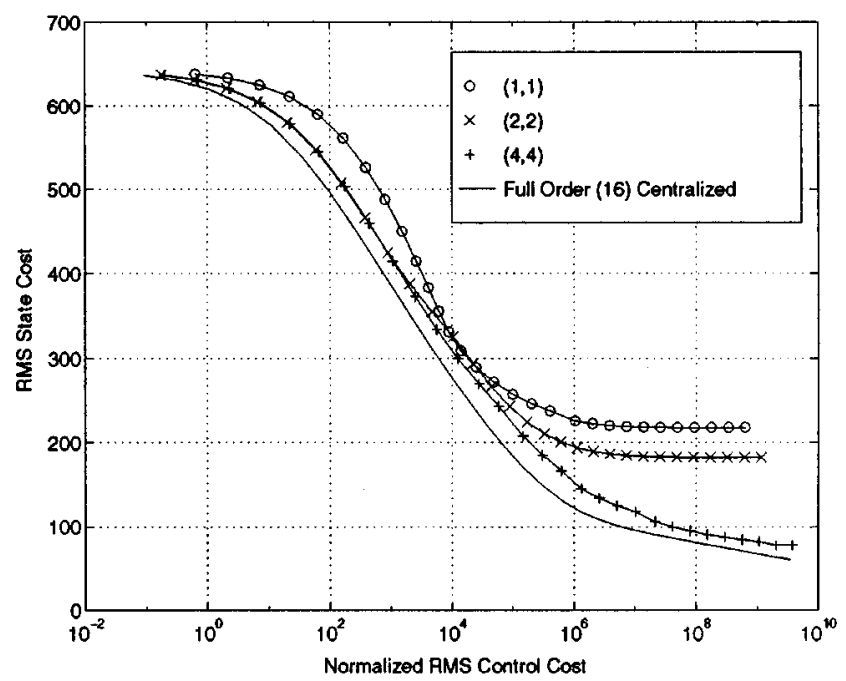

Figure 4. State/control cost trade-off curves for the 16 th-order acoustic duct with decentralized $\mathscr{H}_{2}$-optimal dynamic compensation

for the acoustic duct plant with the same weights as in Section 7.1, using the normal-form parametrization given in Section 3.4. Table I compares the achieved cost, the number of iterations, and the computational effort required for the two parametrizations. The parameter $\sigma$ is a scaling factor used to vary the controller authority (see Appendix). As $\sigma$ decreases, the controller authority increases. Both cases were initialized with the same controller for $\sigma=1$. For the first six values of $\sigma$, Table I shows the full-matrix parametrization converging to an acceptable solution in 
Table I. Full-matrix versus normal-form parametrization computational performance

\begin{tabular}{|c|c|c|c|c|c|c|}
\hline \multirow[b]{2}{*}{$\sigma$} & \multicolumn{3}{|c|}{ Full matrix } & \multicolumn{3}{|c|}{ Normal form } \\
\hline & $\left\|\tilde{G}_{z w}(s)\right\|_{2}^{2}$ & Iterations & CPU seconds & $\left\|\tilde{G}_{z w}(s)\right\|_{2}^{2}$ & Iterations & CPU seconds \\
\hline $1 \cdot 000$ & $636 \cdot 97$ & 226 & $143 \cdot 62$ & $636 \cdot 97$ & 241 & $216 \cdot 68$ \\
\hline $4.714 \times 10^{-1}$ & $615 \cdot 24$ & 171 & $109 \cdot 28$ & $615 \cdot 24$ & 177 & $157 \cdot 32$ \\
\hline $1 \cdot 842 \times 10^{-1}$ & 529.99 & 170 & $109 \cdot 45$ & 529.99 & 179 & $160 \cdot 59$ \\
\hline $7 \cdot 196 \times 10^{-2}$ & 395.60 & 190 & $123 \cdot 84$ & 395.60 & 193 & $173 \cdot 10$ \\
\hline $2 \cdot 811 \times 10^{-2}$ & $278 \cdot 29$ & 181 & $119 \cdot 08$ & $278 \cdot 29$ & 190 & $170 \cdot 26$ \\
\hline $1.098 \times 10^{-2}$ & 201.09 & 209 & $134 \cdot 87$ & 201.09 & 419 & $378 \cdot 85$ \\
\hline $4.291 \times 10^{-3}$ & $149 \cdot 39$ & 338 & $225 \cdot 98$ & $149 \cdot 32$ & 293 & $261 \cdot 57$ \\
\hline $1.676 \times 10^{-3}$ & $130 \cdot 28$ & 551 & $376 \cdot 12$ & $130 \cdot 04$ & 459 & $419 \cdot 38$ \\
\hline $6.551 \times 10^{-4}$ & $119 \cdot 01$ & 332 & $226 \cdot 49$ & 118.95 & 399 & $352 \cdot 30$ \\
\hline $2 \cdot 559 \times 10^{-4}$ & 111.96 & 5000 & $3500 \cdot 12$ & 111.96 & 313 & $286 \cdot 95$ \\
\hline $1.000 \times 10^{-4}$ & $106 \cdot 86$ & 2410 & $1666 \cdot 29$ & $107 \cdot 17$ & 357 & $320 \cdot 06$ \\
\hline
\end{tabular}

fewer iterations, and overall savings in CPU seconds required. For the last four values of $\sigma$, however, the normal-form parametrization requires less computational effort, in some cases providing an order of magnitude saving in total iterations and CPU seconds. Note that the optimal closed-loop cost attained by both parametrizations is the same for the first to sixth and tenth values of $\sigma$. Recalling the discussion in Section 3.4, this implies that the optimal controllers can be represented by the normal-form parametrization for these values of $\sigma$. For the seventh to ninth values, the normal form parametrization actually achieves a lower cost than the full-matrix parametrization. This fact is somewhat counter-intuitive, as the set of controllers that can be represented in the normal-form parametrization is a subset of those that can be represented in the full-matrix parametrization.

\subsection{Centralized and decentralized strictly proper compensation with uncertain plant dynamics}

To demonstrate robust controller synthesis, we considered the 16th-order acoustic duct model with uncertainty in the damped natural frequencies of the third and fourth modes. Both centralized and decentralized strictly proper dynamic compensators were considered. Controllers obtained from the $\mathscr{H}_{2}$-optimal synthesis example considered in Section 7.1 for both structures were used as initial designs for scaled Popov synthesis as discussed in Section 6. Table II gives the predicted and achieved performance and stability regions for the centralized designs while Table III shows the results for the decentralized designs. For performance, the 'Synthesis' column shows the inverse of the value of $\gamma$ used during synthesis, converted to per cent nominal damped natural frequency (DNF), while the 'Numerical' column shows the robust performance region computed by evaluating the actual closed-loop $\mathscr{H}_{2}$ norm over an 2020 point polar grid of variations to determine where the $\mathscr{H}_{2}$ norm rose above the bound given by (81). For stability, the 'Analysis' column is based on the peak $\mu$-bound results given in Reference 12, while the 'Numerical achieved' values were obtained by checking closed-loop stability over a 2020 point polar grid of variations.

\subsection{Centralized and decentralized strictly proper compensation with uncertain input matrices}

As a final example we consider an extension of the problem introduced in Reference 34, involving the two-dimensional rotational and translational dynamics of a rigid beam (Figure 5) 
Table II. Conservatism analysis for centralized compensation with uncertain dynamics matrix

\begin{tabular}{|c|c|c|c|}
\hline \multicolumn{2}{|c|}{ Performance region ( $\%$ nominal DNF) } & \multicolumn{2}{|c|}{ Stability region $(\%$ nominal $\mathrm{DNF})$} \\
\hline Synthesis & Numerical & Analysis & Numerical \\
\hline $0\left(\mathscr{H}_{2}\right.$-optimal $)$ & - & $0 \cdot 2080$ & $0 \cdot 2133$ \\
\hline 0.0008 & $1 \cdot 9556$ & 1.9928 & 1.9971 \\
\hline $0 \cdot 0128$ & $6 \cdot 8862$ & $6 \cdot 8704$ & 6.8903 \\
\hline $0 \cdot 2048$ & $36 \cdot 3316$ & $21 \cdot 7238$ & $36 \cdot 4942$ \\
\hline $3 \cdot 2768$ & $34 \cdot 5717$ & $23 \cdot 7460$ & $34 \cdot 5916$ \\
\hline $23 \cdot 3011$ & $41 \cdot 7615$ & $31 \cdot 8960$ & $41 \cdot 7738$ \\
\hline $63 \cdot 1202$ & $84 \cdot 5715$ & $70 \cdot 1398$ & $84 \cdot 6459$ \\
\hline
\end{tabular}

Table III. Conservatism analysis for decentralized compensation with uncertain dynamics matrix

\begin{tabular}{|c|c|c|c|}
\hline \multicolumn{2}{|c|}{ Performance region ( $\%$ nominal DNF) } & \multicolumn{2}{|c|}{ Stability region ( $\%$ nominal DNF) } \\
\hline Synthesis & Numerical & Analysis & Numerical \\
\hline 0 ( $\mathscr{H}_{2}$-optimal $)$ & - & $0 \cdot 3049$ & $0 \cdot 3101$ \\
\hline 0.0008 & $24 \cdot 2537$ & $17 \cdot 5778$ & $24 \cdot 6619$ \\
\hline $0 \cdot 0128$ & $41 \cdot 3477$ & $33 \cdot 5567$ & $41 \cdot 4834$ \\
\hline $0 \cdot 2048$ & $94 \cdot 3248$ & $72 \cdot 1045$ & $94 \cdot 3370$ \\
\hline $3 \cdot 2768$ & $92 \cdot 4710$ & $51 \cdot 6971$ & $92 \cdot 4864$ \\
\hline $34 \cdot 9513$ & $91 \cdot 4172$ & $59 \cdot 1806$ & $91 \cdot 4251$ \\
\hline
\end{tabular}

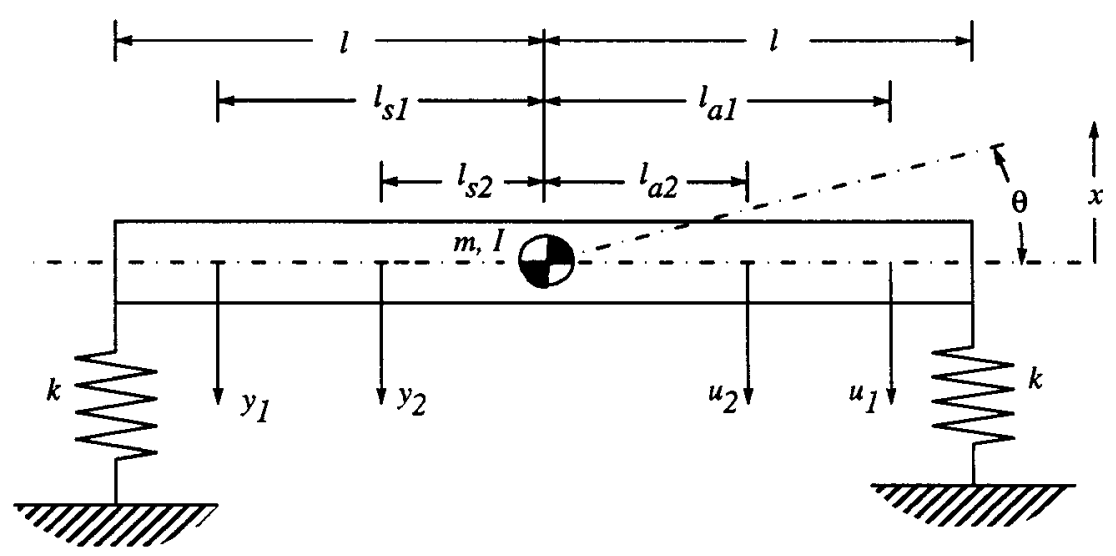

Figure 5. Rigid beam example

with uncertain actuator positions. As shown in the Appendix, uncertain actuator positions correspond to real parameter uncertainty in the matrix $B_{u}$ of the plant. Full-order $\left(n_{\mathrm{c}}=4\right)$ centralized and reduced-order decentralized $\left(n_{\mathrm{c} 1}, n_{\mathrm{c} 2}\right)=(2,2)$ strictly proper dynamic compensators were synthesized for this example. Figure 6 shows the dependence of the closed-loop $\mathscr{H}_{2}$ norm on variations in the second actuator position for centralized designs with the first actuator 


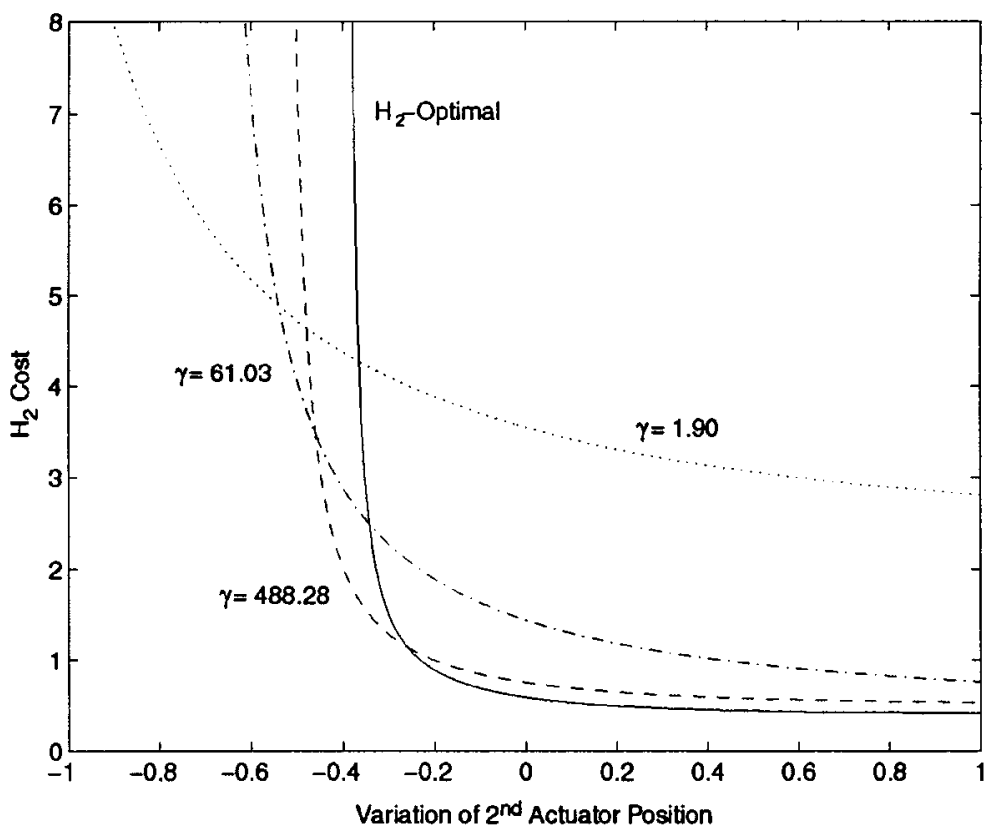

Figure 6. $\mathscr{H}_{2}$ cost versus variation of second actuator non-dimensionalized position for the rigid beam example with full-order $n_{\mathrm{c}}=4$ centralized dynamic compensator

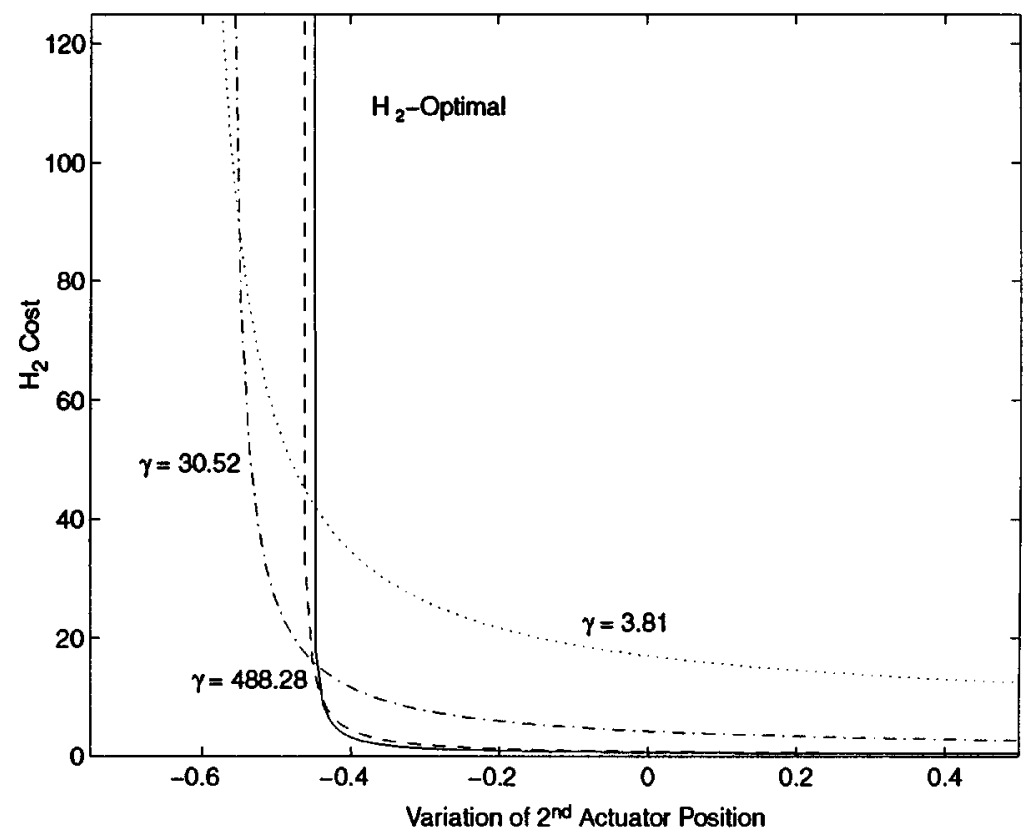

Figure 7. $\mathscr{H}_{2}$ cost versus variation of second actuator non-dimensionalized position for the rigid beam example with reduced-order $\left(n_{\mathrm{c} 1}, n_{\mathrm{c} 2}\right)=(2,2)$ decentralized dynamic compensator 
Table IV. Conservatism analysis of the closed-loop system with uncertain input matrix and centralized compensation

\begin{tabular}{|c|c|c|c|}
\hline \multicolumn{2}{|c|}{ Performance region } & \multicolumn{2}{|c|}{ Stability region } \\
\hline Synthesis & Numerical & Analysis & Numerical \\
\hline $0\left(\mathscr{H}_{2}\right.$-optimal $)$ & - & $0 \cdot 1898$ & $0 \cdot 2684$ \\
\hline $8.0000 \times 10^{-6}$ & $0 \cdot 0103$ & $0 \cdot 1905$ & $0 \cdot 2694$ \\
\hline $1.2800 \times 10^{-4}$ & $0 \cdot 2595$ & $0 \cdot 3966$ & $0 \cdot 5605$ \\
\hline 0.0020 & 0.0725 & $0 \cdot 2638$ & $0 \cdot 3732$ \\
\hline $0 \cdot 0327$ & $0 \cdot 1790$ & $0 \cdot 2245$ & $0 \cdot 3176$ \\
\hline $0 \cdot 5246$ & $0 \cdot 8948$ & $0 \cdot 8175$ & $1 \cdot 1166$ \\
\hline
\end{tabular}

Table V. Conservatism analysis of the closed-loop system with uncertain input matrix and decentralized compensation

\begin{tabular}{|c|c|c|c|}
\hline \multicolumn{2}{|c|}{ Performance region } & \multicolumn{2}{|c|}{ Stability region } \\
\hline Synthesis & Numerical & Analysis & Numerical \\
\hline 0 ( $\mathscr{H}_{2}$-optimal $)$ & - & $0 \cdot 2235$ & $0 \cdot 3161$ \\
\hline $8.0000 \times 10^{-6}$ & $0 \cdot 0033$ & $0 \cdot 2254$ & $0 \cdot 3188$ \\
\hline $1.2800 \times 10^{-4}$ & $0 \cdot 0245$ & $0 \cdot 2116$ & $0 \cdot 2992$ \\
\hline $0 \cdot 0020$ & $0 \cdot 1913$ & $0 \cdot 2301$ & $0 \cdot 3254$ \\
\hline $0 \cdot 0327$ & $0 \cdot 2193$ & $0 \cdot 3079$ & 0.4343 \\
\hline
\end{tabular}

position held at its nominal value. Figure 7 presents the same results for decentralized designs. Comparing the two figures and noting the vertical scale difference, it is apparent that the decentralized controllers require much larger sacrifices of nominal performance for comparable increases in the robust performance region.

Table IV gives the predicted and achieved performance and stability regions for the centralized designs, while Table $\mathrm{V}$ shows the results for the decentralized designs.

\section{DISCUSSION AND CONCLUSIONS}

In this paper we described and illustrated a practical methodology for direct, robust, fixedstructure controller synthesis based upon the decentralized static output feedback formulation for capturing a large class of controller structures. We demonstrated the use of quasi-Newton optimization algorithms for computing optimal gains for centralized and decentralized controllers. We then extended these results to the robust performance problem, demonstrating the use of scaled Popov bounds for synthesizing robust, fixed-structure controllers. Finally, several numerical examples were given to illustrate the methodology and demonstrate the guaranteed and achieved stability and performance of these controllers. Future work includes extending this conservatism analysis to other methods of real-parameter robust controller synthesis for comparison. 


\section{APPENDIX. NUMERICAL EXAMPLES}

\section{A.1. Acoustic duct example 33}

The 16th-order acoustic duct model consists of the dynamic equations

$$
\begin{aligned}
& \dot{x}=A x+B \hat{u}+F_{1} w \\
& \hat{y}=C x+D \hat{u}+F_{2} w \\
& z=E_{1} x+E_{2} \hat{u}+E_{0} w
\end{aligned}
$$

The dynamics matrix is given by $A=\operatorname{block-\operatorname {diag}}\left(A_{1}, \ldots, A_{8}\right)$, where

$$
A_{i}=\left[\begin{array}{cc}
0 & 1 \\
-\omega_{\mathrm{n} i}^{2} & -2 \zeta_{i} \omega_{\mathrm{n} i}
\end{array}\right], \quad \omega_{\mathrm{n} i}=i \pi c L
$$

where $L=10 \cdot 0, c=343 \cdot 0$ and $\zeta_{i}=0 \cdot 01, i=1, \ldots, 8$. For $i=1, \ldots, 16$ and $j=1,2$, the entries of the input and output matrices $B \in \mathscr{R}^{16 \times 2}$ and $C \in \mathscr{R}^{2 \times 16}$ are given by

$$
B(i, j)=\left\{\begin{array}{ccc}
0, & i \text { odd, } \\
c \sqrt{\frac{2}{L}} \sin \frac{i \pi a_{j}}{2 L} & i \text { even, }
\end{array} \quad C(j, i)=\left\{\begin{array}{cl}
c \sqrt{\frac{2}{L} \sin \frac{(i+1) \pi s_{j}}{2 L},} & i \text { odd } \\
0, & i \text { even }
\end{array}\right.\right.
$$

where the positions $s_{1}, s_{2}$ of the sensors and $a_{1}, a_{2}$ of the actuators are

$$
\begin{array}{ll}
a_{1}=4.3750, & s_{1}=0.6250 \\
a_{2}=5.6250, & s_{2}=5.6250
\end{array}
$$

The weighting matrices $F_{2} \in \mathscr{R}^{2 \times 3}, E_{2} \in \mathscr{R}^{3 \times 2}, E_{1} \in \mathscr{R}^{3 \times 16}$ and $F_{1} \in \mathscr{R}^{16 \times 3}$ are given by

$$
\begin{array}{ccc}
E_{2}=\sigma\left[\begin{array}{lll}
0 & 1 & 0 \\
0 & 0 & 1
\end{array}\right]^{\mathrm{T}}, & F_{2}=\tau\left[\begin{array}{ccc}
0 & 1 & 0 \\
0 & 0 & 1
\end{array}\right] \\
F_{1}=\left[\begin{array}{ll}
D_{11} & 0_{16 \times 2}
\end{array}\right], & E_{1}=\left[\begin{array}{c}
E_{11} \\
0_{2 \times 16}
\end{array}\right]
\end{array}
$$

where $\sigma$ and $\tau$ are real numbers, and, for $i=1, \ldots, 16$,

$$
F_{11}(i, 1)=\left\{\begin{array}{cl}
0, & i \text { odd, } \\
c \sqrt{\frac{2}{L} \sin \frac{i \pi h_{1}}{2 L}} & i \text { even, }
\end{array} \quad E_{11}(1, i)=\left\{\begin{array}{cc}
c \sqrt{\frac{2}{L} \sin \frac{(i+1) \pi b_{1}}{2 L},} & i \text { odd } \\
0, & i \text { even }
\end{array}\right.\right.
$$

where the location $h_{1}$ of the disturbance actuator and the location $b_{1}$ of the performance sensor are given by

$$
h_{1}=5 \cdot 6250, \quad b_{1}=9 \cdot 3750
$$

Finally, $D=0_{2 \times 2}$, and $E_{0}=0_{3 \times 3}$.

For robust controller synthesis with uncertainty in the plant dynamics matrix, a similarity transformation was used to transform the plant from the modal form given above to normal form. The similarity transformation is given by

$$
\begin{aligned}
A^{\prime} & =S A S^{-1}, & B^{\prime}=S B, & F_{1}^{\prime}=S F_{1} \\
C^{\prime} & =C S^{-1}, & D^{\prime}=D, & F_{2}^{\prime}=F_{2} \\
E_{1}^{\prime} & =E_{1} S^{-1}, & E_{2}^{\prime}=E_{2}, & E_{0}^{\prime}=E_{0}
\end{aligned}
$$


where $S=$ block-diag $\left(S_{1}, \ldots, S_{8}\right)$, where

$$
S_{i} \triangleq\left[\begin{array}{cc}
\omega_{\mathrm{d} i} & 0 \\
\eta_{i} & 1
\end{array}\right], \quad \omega_{\mathrm{d} i} \triangleq \omega_{\mathrm{n} i} \sqrt{1-\zeta_{i}^{2}}, \quad \eta_{i} \triangleq \omega_{\mathrm{n} i} \zeta_{i}
$$

The uncertain plant is then given by

$$
\begin{aligned}
\dot{x} & =\left(A^{\prime}+M_{A} \Delta N_{A}\right) x+B^{\prime} \hat{u}+F_{1}^{\prime} w \\
\hat{y} & =C^{\prime} x+D^{\prime} \hat{u}+F_{2}^{\prime} w \\
z & =E_{1}^{\prime} x+E_{2}^{\prime} \hat{u}+E_{0}^{\prime} w
\end{aligned}
$$

where

$$
M_{A}=\left[\begin{array}{cc}
M_{13} & M_{14} \\
M_{23} & M_{24} \\
\vdots & \vdots \\
M_{83} & M_{84}
\end{array}\right], \quad N_{A}=\left[\begin{array}{llll}
N_{31} & N_{32} & \cdots & N_{38} \\
N_{41} & N_{42} & \cdots & N_{48}
\end{array}\right]
$$

where

$$
M_{i j} \triangleq\left\{\begin{array} { c c } 
{ 0 _ { 2 \times 2 } } & { i \neq j , } \\
{ [ \begin{array} { c c } 
{ 0 } & { \omega _ { \mathrm { d } j } } \\
{ - \omega _ { \mathrm { d } j } } & { 0 }
\end{array} ] , } & { i = j , }
\end{array} \quad N _ { i j } \triangleq \left\{\begin{array}{ll}
0_{2 \times 2}, & i \neq j \\
I_{2}, & i=j
\end{array}\right.\right.
$$

\section{A.2. Rigid beam example ${ }^{34}$}

The two-dimensional rotational and translational dynamics of the rigid beam shown in Figure 5 with uncertainty in the actuator positions is given by the linear, time-invariant model

$$
\begin{aligned}
\dot{x} & =A x+\left(B+M_{B} \Delta_{B} N_{B}\right) \hat{u}+F_{1} w \\
\hat{y} & =C x+D \hat{u}+F_{2} w \\
z & =E_{1} x+E_{2} \hat{u}+E_{0} w
\end{aligned}
$$

where

$$
\begin{gathered}
A=\left[\begin{array}{cccc}
0 & 1 & 0 & 0 \\
-\frac{2 k}{m} & 0 & 0 & 0 \\
0 & 0 & 0 & 1 \\
0 & 0 & -\frac{2 k l^{2}}{I} & 0
\end{array}\right], \quad B=\left[\begin{array}{cc}
0 & 0 \\
\frac{1}{m} & \frac{1}{m} \\
\frac{l_{a 1}}{I} & \frac{l_{a 2}}{I}
\end{array}\right], \quad C=\left[\begin{array}{cccc}
0 & 1 & 0 & -l_{s 1} \\
0 & 1 & 0 & -l_{s 2}
\end{array}\right] \\
D=\left[\begin{array}{ll}
0 & 0 \\
0 & 0
\end{array}\right], \quad F_{1}=\left[\begin{array}{cccc}
0 & 0 & 0 & 0 \\
\frac{1}{m} & \frac{1}{m} & 0 & 0 \\
0 & 0 & 0 & 0 \\
\frac{l_{a 1}}{I} & \frac{l_{a 2}}{I} & 0 & 0
\end{array}\right], E_{1}=\left[\begin{array}{cccc}
0 & 1 & 0 & -l_{s 1} \\
0 & 1 & 0 & -l_{s 2} \\
0 & 0 & 0 & 0 \\
0 & 0 & 0 & 0
\end{array}\right]
\end{gathered}
$$




$$
\begin{gathered}
F_{2}=\left[\begin{array}{cccc}
0 & 0 & 0 \cdot 1 & 0 \\
0 & 0 & 0 & 0 \cdot 1
\end{array}\right], \quad E_{2}=\left[\begin{array}{cc}
0 & 0 \\
0 & 0 \\
0 \cdot 1 & 0 \\
0 & 0 \cdot 1
\end{array}\right], \quad E_{0}=\left[\begin{array}{llll}
0 & 0 & 0 & 0 \\
0 & 0 & 0 & 0 \\
0 & 0 & 0 & 0 \\
0 & 0 & 0 & 0
\end{array}\right] \\
M_{B}=\left[\begin{array}{cc}
0 & 0 \\
0 & 0 \\
0 & 0 \\
\frac{1}{I} & \frac{1}{I}
\end{array}\right], \quad N_{B}=\left[\begin{array}{ll}
1 & 0 \\
0 & 1
\end{array}\right]
\end{gathered}
$$

and $\Delta$ is given by

$$
\Delta_{B}=\left[\begin{array}{cc}
\delta_{1} & 0 \\
0 & \delta_{2}
\end{array}\right]
$$

where $\delta_{1}$ and $\delta_{2}$ are the deviation of the first and second actuators, respectively, from their nominal positions. The numerical values used are

$$
m=2, I=0 \cdot 5, l=2, k=0 \cdot 25, l_{u 1}=0 \cdot 5, l_{u 2}=1, l_{s 1}=0 \cdot 5, l_{s 2}=1
$$

\section{ACKNOWLEDGEMENTS}

This research was supported by the Air Force Office of Scientific Research under grant F4962095-1-0019, the USAF wright laboratory and the USAF Phillips Laboratory.

\section{REFERENCES}

1. Anderson, B. D. O. and Y. Liu, 'Controller reduction: concepts and approaches', IEEE Trans. Automat. Control, 34, 802-812 (1989).

2. Fuhrmann, P. A. and R. Ober, 'A functional approach to LQG balancing', Int. J. Control, 57, 627-741 (1993).

3. Jonckheere, E. and L. Silverman, 'A new set of invariants for linear systems - application to reduced-order controller design', IEEE Trans. Automat. Control, 28, 953-964 (1983).

4. Hyland, D. C. and D. S. Bernstein, 'Optimal projection equations for fixed-order dynamic compensation', IEEE Trans. Automat. Control, 29, 1034-1037 (1984).

5. Wenk, C. J. and C. H. Knapp, 'Parameter optimization in linear systems with arbitrarily constrained controller structure', IEEE Trans. Automat. Control, 25, 496-500 (1980).

6. Mercadal, M., 'Homotopy approach to optimal, linear quadratic, fixed-architecture compensation', AIAA J. Guid. Contr. Dyn., 14, 1224-1233 (1991).

7. Nett, C. N., D. S. Bernstein and W. M. Haddad, 'Minimal complexity control law synthesis, Part 1: problem formulation and reduction to optimal static output feedback', Proc. Amer. Contr. Conf., Pittsburgh, PA, June 1989, pp. 2056-2064.

8. Bernstein, D. S., W. M. Haddad and C. N. Nett, 'Minimal complexity control law synthesis, part 2: problem solution via $H_{2} / H_{\infty}$ optimal static output feedback', Proc. Amer. Contr. Conf., Pittsburgh, PA, June 1989, pp. 2506-2511. Also appears in Recent Advances in Robust Control, P. Dorato and R. K. Yedavalli (Eds) IEEE Press, 1990, pp. $288-293$.

9. Wang, Y. W. and D. S. Bernstein, ' $H_{2}$ optimal control with an $\alpha$-shifted pole constraint', Int. J. Control, 58, 1201-1214 (1993).

10. Bernstein, D. S., W. M. Haddad and A. G. Sparks, 'A Popov criterion for uncertain linear multivariable systems', Automatica, 31, 1061-1064 (1995).

11. Sparks, A. G. and D. S. Bernstein, 'The scaled Popov criterion and bounds for the real structured singular value', Proc. IEEE Conf. Dec. Contr., Orlando, FL, December 1994, pp. 2998-3002. 
12. Sparks, A. G. and D. S. Bernstein, 'Reliable state space upper bounds for the peak structured singular value', Proc. Amer. Contr. Conf., Seattle, WA, June 1995, pp. 2419-2423. Also Int. J. Robust Nonlin. Control (to appear).

13. Haddad, W. M. and D. S. Bernstein, 'Parameter-dependent Lyapunov functions and the Popov criterion in robust analysis and synthesis', IEEE Trans. Automat. Control, 40, 536-543 (1995).

14. Sparks, A. G. and D. S. Bernstein, 'Real structured singular value synthesis using the scaled Popov criterion', $A I A A$ J. Guid. Contr. Dyn., 18, 1244-1252 (1995).

15. Fan, M., A. Tits and J. Doyle, 'Robustness in the presence of mixed parametric uncertainty and unmodelled dynamics', IEEE Trans. Automat. Control, 36, 25-38 (1991).

16. Packard, A. and J. Doyle, 'The complex structured singular value', Automatica, 29, 71-109 (1993).

17. Badawi, F. A., 'On a quadratic matrix inequality and the corresponding algebraic Riccati equation', Int. J. Control, 36, 313-322 (1982).

18. Nesterov, Y. and A. Nemirovskii, Interior-point Polynomial Methods in Convex Programming, volume 13 of Studies in Applied Mathematics, SIAM, 1994

19. Boyd, S., L. El Chaoui, E. Feron and V. Balakrishnan, Linear Matrix Inequalities in Systems and Control Theory, volume 15 of Studies in Applied Mathematics, SIAM, 1994.

20. Gahinet, P., A. Nemirovskii, A. J. Laub and M. Chilali, LMILab: A Package for Manipulating and Solving LMIs, v. 2.0, The Math Works, Inc., 1994.

21. Dennis, J. E., Jr. and R. B. Schnabel, Numerical Methods for Unconstrained Optimization and Nonlinear Equations, Prentice-Hall, 1983.

22. Khaner, D., C. Moler and S. Nash, Numerical Methods and Software, Prentice-Hall, 1988.

23. Mäkilä, P. M. and H. T. Toivonen, 'Computational methods for parametric LQ problems - a survey', IEEE Trans. Automat. Control, 32, 658-671 (1987).

24. Seinfeld, D. R., W. M. Haddad, D. S. Bernstein and C. N. Nett, ' $H_{2} / H_{\infty}$ controller synthesis: illustrative numerical results via quasi-Newton methods', Proc. Amer. Contr. Conf., Boston, MA, June 1991, pp. 1155-1156.

25. Harn, Y. and R. L. Kosut, 'Optimal low-order controller design via 'LQG-like' parametrization', Automatica, 29, 1377-1394 (1993).

26. Ullauri, J., D. E. Walker and D. B. Ridgely, 'Reduced order mixed $H_{2} / H_{\infty}$ optimization with multiple $H_{\infty}$ constraints', Proc. AIAA Guid., Nav. Contr. Conf., Scottsdale, AZ, June 1994, pp. 1051-1060.

27. Hyland, D. C. and S. Richter, 'On direct versus indirect methods for reduced-order controller design', IEEE Trans. Automat. Control, 35, 377-379 (1990).

28. Ge, Y., L. T. Watson, R. S. Erwin and D. S. Bernstein, 'Globally convergent homotopy algorithms for full-order LQG control with an $H_{\infty}$ performance bound', Proc. Amer. Contr. Conf., Baltimore, MD, June 1994, pp. 1906-1910.

29. Ge, Y., L. T. Watson, E. G. Collins Jr. and D. S. Bernstein, 'Probability-one homotopy algorithms for full- and reduced-order $\mathrm{H}_{2} / \mathrm{H}_{\infty}$ controller synthesis', Proc. IEEE Conf. Dec. Contr., Orlando, FL, December 1994, pp. 2672-2677.

30. Safonov, M. G., K. C. Goh and J. H. Ly, 'Control system synthesis via bilinear matrix inequalities', Proc. Amer. Contr. Conf., Baltimore, MD, June 1994, pp. 45-49.

31. Bernstein, D. S., W. M. Haddad and A. G. Sparks, 'A simplified proof of the multivariable Popov criterion and an upper bound for the structured singular value for real parameter uncertainty', Proc. IEEE Conf. Dec. Contr., Orlando, FL, December 1994, pp. 2139-2140.

32. Collins, E. G., Jr., W. M. Haddad and S. S. Ying, 'Nearly non-minimal linear-quadratic-Gaussian compensators for reduced-order control design initialization', AIAA J. Guid. Contr. Dyn., 19, 259-261 (1996).

33. Hong, J. et al., 'Modeling, identification, and feedback control of noise in an acoustic duct', IEEE Trans. Contr. Sys. Tech., 4, 283-291 (1996).

34. Doyle, J. C., B. A. Francis and A. R. Tannenbaum, Feedback Control Theory, Macmillan, New York, 1992. 